\title{
Edítoríal.
}

\section{THE LOCALIZATION OF INTRACRANIAL TUMOURS.}

UP to the year 1870 almost nothing was known of cerebral localization of function, the generally accepted view being that of Flourens, who taught that the cerebrum is a homogeneous mass, and that symptoms resulting from cerebral lesions are proportional to the amount of brain tissue lost. An entirely new field of study was inaugurated by the chance observations of an army surgeon on the battle-field. Fritsch, whilst operating upon a wounded soldier, applied the galvanic current to the exposed surface of the brain, and observed twitching of certain muscles. Further pioneer work, undertaken in collaboration with Hitzig, furnished conclusive proof of the error of the view then prevalent that the cerebral hemispheres are not excitable by any known stimulus. Their discovery was immediately followed by an immense number of experimental researches which in general have given concordant results, and the fruitful labours of Horsley, Ferrier, Sherrington, and a host of other workers have made the subject of brain function so familiar that to-day it is difficult to realize what a revolution in medicine cerebral localization has made. Although the widely accepted view that particular functions are localized in certain definite cortical 'centres' cannot withstand criticism, the acceptance of a cerebral localization of symptoms has proved of great clinical value, and been responsible for much progress in the surgery of the nervous system. It needs but a small acquaintance with the literature to realize the rapid growth of brain surgery in the last fifty years, especially in relation to operative procedures for the removal of intracranial neoplasms.

Decompressions in cases of brain tumour were at first purely palliative in character, the trephine being employed solely for the relief of pressure or other urgent symptoms. The honour of being the first to open the skull as a palliative procedure is shared by Horsley and Durante, although Annandale in 1894 stated that more than twenty years previously he had trephined the skull of a patient whose symptoms were those of general brain pressure. 
With the development of improved methods of technique the surgeon next ventured to undertake an operation which aimed at the removal of the neoplasm, provided it was situated in the more accessible regions of the hemisphere. The early results were disheartening, and served to damp the enthusiasm excited by the application of cerebral localization to surgery. In recent years, however, newer methods of approach and earlier interference have done much to reduce the high case-mortality, and at the present day tumours are successfully removed from almost every part of the brain. Indeed, it would almost appear that we have reached a phase in which operative procedures are in advance of the methods of diagnosis. It seems very nearly incredible that an intracranial neoplasm may grow to the size of one's fist and yet evade accurate localization; but probably few neurologists have escaped the chagrin of observing such cases. All that it may be possible to say in the absence of focal symptoms is that a tumour is situated in one or other hemisphere, or, more vaguely still, that it is confined to the supratentorial region. It is particularly when the growth infiltrates or destroys one of the many 'silent' areas of the brain that difficulties arise. Moreover, the physician is occasionally misled by a remarkable absence of signs of localizing value when the tumour occupies an area whose function is known. Still another source of complexity arises when local signs are late in making their appearance, or when the patient is seen for the first time at a late stage of his illness, for, under such conditions, symptoms due to derangement of function in parts of the brain at a distance from the tumour may suggest a localization which in no way reflects the true state of affairs.

It follows from what has just been said, that while the presence of an intracranial tumour can usually be readily diagnosed it may be difficult or impossible to determine its exact locality. This occasional failure of ordinary neurological methods has naturally led workers to seek other means of approach. Puncture and aspiration of the brain through the skull was practised many years ago; but inasmuch as one puncture seldom sufficed, the procedure has been abandoned. The use of the tuning-fork has also found adherents. The presence of a tumour close beneath the skull-cap lessens bone conductivity, and if the vertex is struck by a tuning-fork its note may fail to be conducted in the neighbourhood of the lesion. The value of the Röntgen ray for diagnostic purposes has also proved disappointing, it being the exception rather than the rule for a tumour to cast an abnormal shadow, and few surgeons would care to risk a diagnosis upon its indications.

Quite recently, however, it has been found possible to utilize radiography in a manner which we imagine will be new to many of 
our readers. For the purpose of localizing or eliminating tumours in areas above the tentorium cerebelli, Dandy ${ }^{1}$ has employed the Röntgen ray after the fluid contents of the cerebral ventricles have been removed and replaced by air. The introduction of air allows a very clear outline of the cerebral ventricles to be obtained, and it is, of course, evident that a tumour of any size situated in either cerebral hemisphere will modify the shape, size, and position of the corresponding lateral ventricle. The method, which Dandy calls 'ventriculography', has been employed by its inventor in more than 75 cases, and in 5 instances the 'ventriculogram' allowed precise localization of the tumour in the absence of all other localizing signs. In one instructive case in which two exploratory operations had failed to reveal the tumour, $x$-ray examination after an intraventricular injection of air permitted accurate localization. The anterior horn and the anterior portion of the body of the left lateral ventricle were almost exactly like the corresponding parts of the right ventricle, but no air had reached the posterior and descending horns; these portions of the left ventricle, therefore, threw no shadows and were absent in the röntgenogram. Craniotomy was performed, and a large encapsulated tumour removed from the ependymal lining of the left descending horn. Two years after the operation the patient was perfectly well and at work.

This method of localization possesses the disadvantage that it involves the necessity of making a preliminary opening in the skull to allow the introduction of air, and the formidable risks which at present attend its use do not permit its recommendation as a routine procedure. Nevertheless, the available evidence appears to justify the conclusion that ventriculography possesses great possibilities of clinical application, and places in the hands of the ncurologist a new aid to diagnosis which promises still more brilliant achievements in the field of neurological surgery.

REFERENCE.

1DANDY, W. E., " Localization or Elimination of Cerebral Tumours by Ventriculography", Surg. Gynecol. and Obst., 1920, xxx, 329. 